This item was submitted to Loughborough's Research Repository by the author.

Items in Figshare are protected by copyright, with all rights reserved, unless otherwise indicated.

\title{
Mobility of a semi-infinite beam with constant curvature
}

PLEASE CITE THE PUBLISHED VERSION

PUBLISHER

(C) Academic Press / Elsevier

VERSION

AM (Accepted Manuscript)

LICENCE

CC BY-NC-ND 4.0

REPOSITORY RECORD

Walsh, Stephen J., and R.G. White. 2010. "Mobility of a Semi-infinite Beam with Constant Curvature". figshare. https://hdl.handle.net/2134/6488. 
This item was submitted to Loughborough's Institutional Repository (https://dspace.lboro.ac.uk/) by the author and is made available under the following Creative Commons Licence conditions.

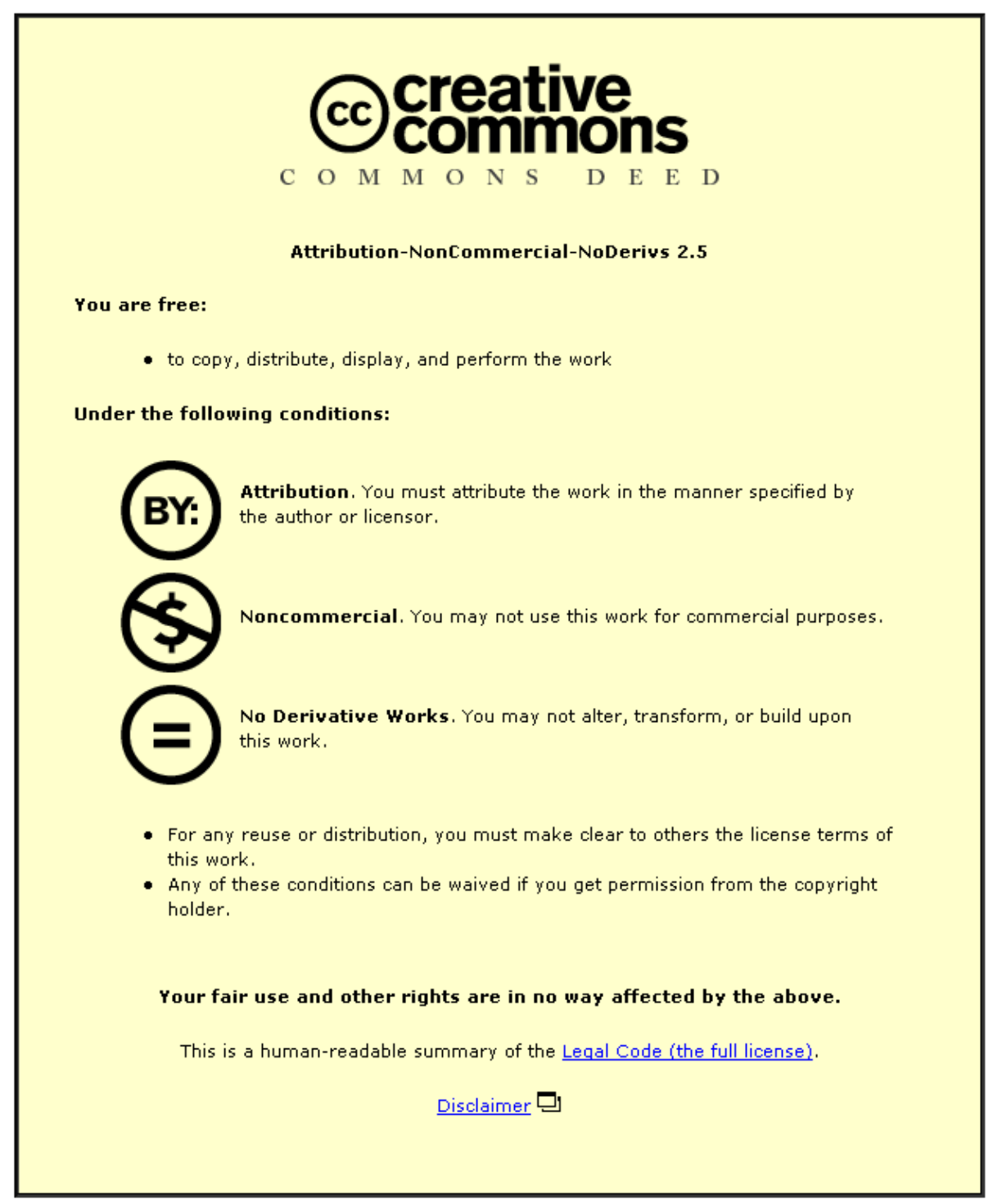

For the full text of this licence, please go to: http://creativecommons.org/licenses/by-nc-nd/2.5/ 
MOBILITY OF A SEMI-INFINITE BEAM WITH CONSTANT CURVATURE

SJ Walsh(1) and RG White (2)

(1) Department of Aeronautical \& Automotive Engineering and Transport Studies Loughborough University, Loughborough, Leics. LE11 3TU. UK

(2) Department of Aeronautics and Astronautics

University of Southampton, Southampton, Hants. SO17 1BJ. UK

4 copies submitted

17 manuscript pages

7 figures

1 tables 
Running headline : Beam with constant curvature

Address for correspondence : Department of AAETS

Loughborough University, Loughborough,

Leicestershire. LE11 3TU 


\section{ABSTRACT}

In a straight beam the flexural and longitudinal wave motions are uncoupled. For a curved beam, however, there is interaction between the longitudinal and bending deformations leading to coupled extensional-flexural wave propagation. In this paper coupled extensional-flexural wave propagation is investigated by considering the mobility of a 'semi-infinite' beam with a constant radius of curvature. Both theoretical and experimental results are discussed and formulae for the point and cross mobilities of the structure are presented. 


\section{INTRODUCTION}

In the design stage of a vehicle there is often a need to predict the vibration transmission to the structure by an applied force. One design method is based upon using formulae for the mobility of simple structural elements. Many useful formulae have already been published for structural elements such as beams, plates and rings. In this paper simple design formulae are sought to describe the mobility of a 'semi-infinite' beam where the centre line forms a plane of constant radius of curvature. The cross-section of the beam is uniform and symmetrical about the plane and it is assumed that there is no motion perpendicular to the plane. It is also assumed that the beam material is linearly elastic, homogeous, isotropic and continuous.

There have been a number of previous studies of the wave motion in a curved beam. In an elementary theory [1] by Love it is , assumed that the centre-line remains unextended during flexural motion, whilst flexural behaviour is ignored when considering extensional motion. Using these assumptions the vibrational behaviour of complete or incomplete rings has been considered by many researchers who are interested in the low frequency behaviour of arches and reinforcing rings [2]. In the same reference [1] Love presented equations for thin shells which include the effects of extension of the mid-surface during bending motion. Flügge [3] has also derived equations for thin shells which include extension of the midsurface during bending motion but which are more consistent when discarding higher order terms. Both these sets of equations can be reduced to equations applicable to a curved beam. Equations derived specifically for a curved beam are presented by Philipson [4] who included extension of the central line in the flexural wave motion, and also rotary inertia effects. In a development analogous to that of Timoshenko for straight beams, Morley [5] introduced a correction for radial shear when considering the vibration of curved beams. Graff later presented frequency versus wave number and wave speed versus wave number data for wave motion in a curved beam, for the Love based equations [6], and when including higher order effects [7].

However, dispersion curves only describe the possible types of wave motion in the structure : no assumpions are made about any excitation force or boundary conditions. In the present work the vibrational response of a curved 'semi-infinite' beam when excited at its free end by a purely circumferential force is developed by considering the propagating and evanescent waves in the beam. This method has previously been used to analyse the flexural response of a straight Euler-Bernoulli beam on periodic [8] and non-periodic supports [9]. To validate the theoretical predictions, experimental studies were undertaken on a curved mild steel beam with a constant radius of curvature. In section three the 
apparatus used for these experiments is described and the measurement method outlined. In section four a comparison between the measured and predicted mobilities is presented which leads to a set of simple formulae for the point and cross mobilities of the structure.

\section{THEORY}

\subsection{BEAM EQUATIONS AND FREE WAVE SOLUTION}

Consider a portion of a curved beam, as shown in Figure 1. The circumferential co-ordinate measured around the centre-line is $\mathrm{s}$, while the outward pointing normal co-ordinate from the centre-line is $\mathrm{z}$, and the general radial co-ordinate is $r$. The centre-line is defined as the locus of centroids of each crosssectional element. The tangential and radial displacements of a material point are $U(r, s, t)$ and $W(r, s, t)$, respectively. For small displacements of thin beams the assumptions known as 'Love's first approximation'[3], in classical shell theory, can be made which leads to the following linear relationships between the displacements of a material point and components of displacement at the undeformed centre-line:

$$
\begin{gathered}
U(r, s, t)=u(R, s, t)+z \phi(s, t), \\
W(r, s, t)=w(R, s, t),
\end{gathered}
$$

where $u$ and $w$ are the components of displacement at the centre-line in the tangential and radial directions, respectively and, $\phi$ is the rotation of the normal to the centre-line during deformation:

$$
\begin{gathered}
\phi=\frac{\mathrm{u}}{\mathrm{R}}-\frac{\partial w}{\partial \mathrm{s}}, \\
\left(\begin{array}{c}
\text { angle of } \\
\text { curvature }
\end{array}\right)\left(\begin{array}{c}
\text { rotational displacement } \\
\text { of straight beam }
\end{array}\right)
\end{gathered}
$$

and $W$ is independent of $z$ and is completely defined by the centre-line component $w$. The relation for total circumferential strain is:

$$
e_{S}=\frac{1}{\left(1+\frac{z}{R}\right)}\left(\varepsilon_{S}+z \beta_{S}\right)
$$

where the in-plane (extensional) strain is given by

$$
\begin{gathered}
\varepsilon_{S}=\underset{\frac{w}{R}}{\left(\begin{array}{c}
\text { stretching due to } \\
\text { radial displacement }
\end{array}\right)}+\frac{\frac{\partial u}{\partial s},}{\left.\begin{array}{c}
\text { stretching due to motion } \\
\text { in circumferential direction }
\end{array}\right)}
\end{gathered}
$$


and the bending strain (mid-surface change in curvature) is given by

$$
\begin{gathered}
\beta_{s}=\frac{\partial \phi}{\partial s}, \\
=\frac{\partial}{\partial s}\left(\frac{\mu}{R}-\frac{\partial w}{\partial s}\right) .
\end{gathered}
$$

See Appendix B for a complete derivation of the strain displacement expression. Since the radial stress component $\sigma_{r}$ is assumed negligible, the transverse strain $\varepsilon_{r}$ is zero, and as a consequence of Kirchoff's hypothesis the transverse shear strain $\gamma_{s r}$ is zero. Assuming the material to be linearly elastic, the circumferential stress-strain relationship is given by Hooke's Law:

$$
\sigma_{\mathrm{S}}=\mathrm{E} \varepsilon_{\mathrm{S}}
$$

where $E$ is Young's modulus. Assuming the material to be homogeneous and isotropic, the material properties $E, G$ and $v$ can be treated as constants. Thus, by integrating the stresses over the beam thickness, force and moment resultants are obtained. Using the strain-displacement expression, equation (4), the circumferential force is given by

$$
N=E S\left(\frac{w}{R}+\frac{\partial u}{\partial s}\right)+\frac{E l}{R}\left(\frac{w}{R^{2}}+\frac{\partial^{2} w}{\partial s^{2}}\right)
$$

where $S$ is the cross-sectional area of the beam, and $I$ is the second moment of area of the crosssection. The bending moment is given by

$$
M=-E l\left(\frac{W}{R^{2}}+\frac{\partial^{2} w}{\partial s^{2}}\right)
$$

Although the transverse shear stress $\sigma_{\mathrm{sr}}$ is zero, a non-vanishing shear resultant, $Q$, is defined as the integral across the thickness of the transverse shear stress, which leads to the following expression:

$$
Q=-E I \frac{\partial}{\partial s}\left(\frac{W}{R^{2}}+\frac{\partial^{2} w}{\partial s^{2}}\right)
$$

Figure 2 shows the sign convention of force resultants on an elemental slice of a curved beam.

Flügge based equations of motion for a curved beam can be obtained by a reduction of the equations of motion for a circular cylindrical shell presented in [3]. This leads to the following set of equations:

$$
R E\left(\frac{\partial^{2} u}{\partial s^{2}}+\frac{1}{R} \frac{\partial w}{\partial s}\right)=\rho R \frac{\partial^{2} u}{\partial t^{2}}
$$




$$
-E K^{2} R \frac{\partial}{\partial s^{2}}\left(\frac{w}{R^{2}}+\frac{\partial^{2} w}{\partial s^{2}}\right)-\left[E\left(\frac{\partial u}{\partial s}+\frac{w}{R}\right)+\frac{E K^{2}}{R}\left(\frac{w}{R^{2}}+\frac{\partial^{2} w}{\partial s^{2}}\right)\right]=\rho R \frac{\partial^{2} w}{\partial t^{2}}
$$

where $\rho$ is the density of the material, and $K$ the radius of gyration. To obtain an harmonic solution assume that flexural and extensional sinusoidal waves propagate in the positive circumferential direction and can be represented respectively by:

$$
\begin{aligned}
& w(s, t)=A \exp [i(\omega t-k s)], \\
& u(s, t)=B \exp [i(\omega t-k s)]
\end{aligned}
$$

Where $A$ and $B$ are the complex wave amplitudes. Substituting these expressions into the equations of motion (12) and (13) gives the harmonic form of the equations of motion:

$$
\begin{gathered}
\left\lfloor\frac{\left[k^{2}\right.}{\rho}-\omega^{2}\right\rfloor B+i \frac{k E}{R \rho} A=0 \\
-\frac{E k k}{R \rho} B+\left\lceil\left(k^{2}-\frac{1}{R^{2}}\right)^{2} \frac{E K^{2}}{\rho}+\frac{E}{R^{2} \rho}-\omega^{2}\right\rfloor A=0 .
\end{gathered}
$$

\subsection{NUMERICAL EXAMPLE}

For a given radian frequency, $\omega$, the harmonic equations of motion were solved to find the complex valued wave number, $k$, and corresponding wave amplitude ratio $B / A$. The physical dimensions and material properties were chosen to be the same as the mild steel beam used for the laboratory experiments. The physical properties of the beam are listed in Table 1. Wave number versus frequency curves for the beam are shown in Figure 3. The frequency range is expressed in terms of the nondimensional frequency $\Omega=\omega \mathrm{R} / \mathrm{c}_{0}$, where $\mathrm{c}_{0}$ is the phase velocity of extensional waves in a straight bar, whilst the wave number range is expressed in terms of the non-dimensional wave number $\mathrm{kR}$. The frequency axis extends over the non-dimensional frequency range $\Omega=0.01$ to 10.0 which represents a dimensional frequency of 8.2 to $8200 \mathrm{~Hz}$. It can be seen in Figure 3 that there are two different frequency regimes separated by the ring frequency $\Omega=1$. Above the ring frequency three wave types are: i) a predominantly flexural travelling wave (marked with the symbol ${ }^{* *}$ ); ii) a predominantly flexural near field wave (marked with the symbol 'o'); and iii) a predominantly extensional travelling wave (marked with the symbol ' + '). It can be seen in Figure 3 that above the ring frequency both flexural travelling waves and flexural near-field waves have the same wave number. Below the ring frequency the predominantly flexural 
travelling wave and the predominantly flexural near field waves still exist, however the predominantly extensional travelling wave is now replaced by a predominantly extensional near field wave (marked with the symbol ' $x$ '). Below $\Omega=0.1$ the predominantly flexural travelling and near field wave numbers diverge, with the travelling wave having the higher wave number.

\subsection{RESPONSE DUE TO CIRCUMFERENTIAL EXCITATION AT THE FREE END}

Assume that a "semi-infinite" beam with curvature is excited at its end by a point harmonic force, $F_{S} e^{i \omega t}$, acting in the circumferential direction as shown in Figure 4. At a given position along the beam the total flexural or extensional displacement will be given by the sum of the displacements of the individual waves travelling away from the end of the beam. Thus, the total flexural displacement is given by

$$
w(s)=A_{1} e^{-k_{1} s}+A_{2} e^{-k_{2} s}+A_{3} e^{-k_{3} s}
$$

where $A_{1}, A_{2}$ and $A_{3}$ represent the unknown wave amplitudes and $k_{1}, k_{2}$ and $k_{3}$ the respective wave numbers. For clarity of notation the harmonic term $\mathrm{e}^{\mathrm{i} \omega t}$ has been omitted. The corresponding extensional displacement is given by

$$
u(s)=B_{1} e^{-k k_{1} s}+B_{2} e^{-k_{2} s}+B_{3} e^{-k_{3} s} .
$$

To determine the response of the structure the boundary conditions and applied load at the free end of the beam need to be evaluated. The boundary conditions and applied loads at $s=0$ are: i) that the axial force resultant is equal to the externally applied force; ii) that the bending moment is zero; and iii) that the shear force is zero. Substituting the displacement equations (18) and (19) into the Flügge based expressions for the resultant forces gives a set of three simultaneous equations in the unknown wave amplitudes $A_{1}, A_{2}$ and $A_{3}$. For a given excitation frequency, $\omega$, the Flügge based equations of motion can be solved to find the three possible wave numbers, $k_{1}, k_{2}$ and $k_{3}$ and the respective extensional to flexural wave amplitude ratios, $B_{j} / A_{j}$. Assuming a 'semi-infinite' beam with waves travelling away from the excitation at the free end, then only three wave amplitudes $\left(A_{1}: A_{2}: A_{3}\right)$ remain unknown. Substituting the previously calculated wave numbers, $k_{i}$ into the set of three simultaneous equations and assuming unit force $F_{s}=1$, enables the unknown wave amplitude ratios $\left(A_{1}: A_{2}: A_{3}\right)$ to be evaluated. The cross 
receptance can now be calculated by evaluating the flexural displacement (equation (1)) at the excitation location:

$$
\alpha_{z F}=\frac{w(0)}{F}=\frac{A_{1}+A_{2}+A_{3}}{F}
$$

Using the extensional to flexural wave amplitude ratios $B_{j} / A_{i}$, the point receptance can be calculated by evaluating the extensional displacement at the excitation location:

$$
\alpha_{s F}=\frac{u(0)}{F}=\frac{B_{1}+B_{2}+B_{3}}{F}
$$

\section{EXPERIMENTAL APPARATUS AND METHOD}

Figure 5 shows a schematic representation of the experimental curved beam. To obtain free conditions at the end of the beam, the whole beam was suspended on thin wires at $1 \mathrm{~m}$ intervals from underneath laboratory benches. It was assumed that no motion would occur in the plane of the suspension system. To obtain "semi-infinite" conditions one end of the beam was inserted into an anechoic termination. This termination consisted of a $1 \mathrm{~m}$ long box filled with sand to dissipate the energy of the wave motion. The excitation force was obtained striking the beam with an instrumented hammer (BK8202) at the free end. To assist in obtaining a purely circumferential force a hard steel triangular block was glued at the centre of the cross-section of the free end of the beam. The applied force was measured with the instrumented hammer, whilst the response acceleration was measured using two 11 gram accelerometers (BK4371). Extensional motion was measured using an accelerometer mounted on the cross-section of the beam just above the triangular block and, flexural motion was measured using an accelerometer, mounted on the centre-line of the side of the beam. The applied force and resulting accelerations were recorded simultaneously on an HP3566A spectrum analyser and the point and cross mobility calculated directly from a single measurement by dividing Fourier transform of the response velocity by that of the applied force.

The instrumented hammer provides an excitation force with a significant frequency spectrum up to approximately $10 \mathrm{k} \mathrm{Hz}$ with a usable frequency range (below the first zero) up to approximately $3 \mathrm{k} \mathrm{Hz}$. Thus, for the frequency response measurements anti-alias filters within the spectrum analyser were set at $3200 \mathrm{~Hz}$, and a sampling rate of 8192 samples/sec chosen, giving a folding frequency of $4096 \mathrm{~Hz}$. To record the entire decaying response a Fast Fourier Transform (FFT) block size of 8192 points was chosen, 
giving a record length of 1 second, and a frequency resolution of $1 \mathrm{~Hz}$. The digitised time histories were transformed to the frequency domain using an FFT algorithm within the spectrum analyser. Since the time histories were recordings of transient signals, the rectangular data window was chosen. The spectra of the acceleration signals were integrated in the frequency domain by division by $i \omega$, and the point and cross mobilities calculated from a single measurement by complex division of the respective velocity spectra by the excitation force spectrum. The mobility data shown in Figures 6 and 7 are presented on nondimensional frequency axes. These were obtained from the dimensional spectra by multiplying the frequency axis by $\frac{2 \pi R}{c_{0}}$.

\section{RESULTS}

The predicted point mobility is compared with the measured point mobility in Figure 6 over the nondimensional frequency range $\Omega=0.01$ to $\Omega=10.0$. Figure $6(\mathrm{a})$ shows the modulus of the point mobility which indicates that there are two frequency regions separated by the ring frequency $\Omega=1.0$. Above the ring frequency the predicted value of the point mobility (marked with 'o' symbols) asymptotes to a constant value of $8 \times 10^{-5}(\mathrm{~m} / \mathrm{s}) / \mathrm{N}$ as the frequency increases. This value corresponds to the point mobility of purely extensional waves in a 'semi-infinite' straight bar given by [10] as

$$
Y_{\mathrm{s}} F=\frac{c_{0}}{E S}
$$

The corresponding measured data show resonant behaviour, with the resonant frequencies corresponding approximately to those of purely extensional waves in a straight rod of length $5 \mathrm{~m}$, with the same material properties as the experimental curved beam.

Below the ring frequency extensional near-field waves are to be expected rather than travelling extensional waves This is confirmed by inspection of the modulus of the point mobility shown in Figure 6(a) where it can be seen that the experimental data do not exhibit resonant behaviour. Both the measured and predicted data exhibit the characteristics of a 'mass line' and it is shown in Appendix A that below the ring frequency the beam acts as a mass of length equal to the radius of curvature, $R$. Further, in terms of the non-dimensional frequency, $\Omega$, the point mobility can be expressed as

$$
Y_{S F}=\frac{-i}{\Omega S \rho c_{0}}
$$


Figure $6(b)$ shows the corresponding phase angle which indicates that below the ring frequency the velocity is $90^{\circ}$ out of phase with force and thus only near field waves are being generated. Above the ring frequency the velocity is in phase with the applied force and, thus travelling waves are being generated.

Figure 7 shows the measured and predicted cross mobility (flexural velocity per unit circumferential force). The modulus is shown in Figure 7 (a) where the measured data indicate resonant behaviour with the measured resonant frequencies corresponding approximately to the natural frequencies due to flexural waves in a free-free beam of length $5 \mathrm{~m}$. This is surprising as the point mobility indicated that the response consists largely of near field extensional waves below the ring frequency, and thus the measured data should not exhibit resonant behaviour. This discrepancy may be due to the wave length of the extensional near field waves being greater than the length of the experimental beam $(5 \mathrm{~m})$. Thus, at the end of the beam within the anechoic termination the predominantly extensional waves may be converted into predominantly flexural travelling waves which gives rise to the resonant behaviour shown in Figure 7. Comparison of the predicted modulus of the cross mobility shown in Figure 7(a) with the predicted modulus of point mobility shown in Figure 6(a) indicates that both functions have the same value below the ring frequency. Comparison of the phase angle of the cross mobility shown in Figure $7(\mathrm{~b})$ with the phase angle of the point mobility shown in Figure $6(\mathrm{~b})$ indicates a $180^{\circ}$ phase difference between the phase angle of the cross mobility and the phase angle of the point mobility below the ring frequency. Thus, from equation (23) the cross mobility is given by

$$
Y_{Z F}=\frac{i}{\Omega S \rho c_{o}}
$$

In terms of radian frequency, $\omega$, this becomes

$$
Y_{\mathrm{ZF}}=\frac{\mathrm{i}}{\omega \mathrm{RS \rho}}
$$

Thus, from equation (25) it can be seen that the cross mobility is dependent upon the frequency, $\omega$, the radius of curvature, $R$, and the mass per unit length, $S \rho$, of the beam. It can also be seen that the flexural velocity is $90^{\circ}$ out of phase with the applied circumferential force.

\section{SUMMARY}

In this paper both measured and predicted levels of the mobility of a 'semi-infinite' beam with a constant radius of curvature have been presented, the beam being excited in the circumferential direction at the free end. The results of this study can be summarised as follows: a) the point mobility has two frequency 
regions separated by the ring frequency. Above the ring frequency the point mobility is dominated by predominantly extensional travelling waves and has a constant value which asymptotes to the point mobility of purely extensional waves in a straight 'semi-infinite' bar. Below the ring frequency the point mobility is dominated by predominatly extensional near field waves and the beam acts as a mass of length equal to the radius of curvature. b) the cross mobility is dependant upon frequency, the radius of curvature and the mass per unit length of the beam. The flexural velocity is $90^{\circ}$ out of phase with the applied circumferential force.

\section{ACKNOWLEDGEMENT}

The experimental work was undertaken at the Institute of Sound and Vibration Research, University of Southampton. 


\section{REFERENCES}

A.E.H. LOVE 1940 A treatise on the mathematical theory of elasticity. Dover Publications, New-York T.E. LANG 1962 Jet Propulsion Laboratory Technical Report, 32-261. Vibration of thin circular rings. A.W. LEISSA 1977 Vibrations of shells. NASA SP-288, Washington D.C.

L.L. PHILIPSON 1956 Journal of Applied Mechanics, 23, 364-366. One the role of extension in the flexural vibrations of rings.

L.S.D. MORLEY 1961 Quarterly Journal of Mechanics and Applied Mathematics, 14 (2), 155-172. Elastic waves in a naturally curved rod.

K.F. GRAFF 1975 Wave motion in elastic solids. Clarendon Press, Oxford.

K.F. GRAFF 1970 IEEE Transactions of Sonics and Ultrasonics, SU-17 (1), 1-16. Elastic wave propagation in a curved sonic transmission line.

D.J. MEAD 1986 Journal of Sound and Vibration, 104, 9-27. A new method of analysing wave motion in periodic structures: Applications to periodic Timoshenko beams and stiffened plates.

9 D.J. MEAD 1990 Journal of Sound and Vibration, 141 (3), 465-484. The harmonic response of uniform beams on multiple linear supports: A flexural wave analysis.

L. CREMER, M HECKL AND EE UNGAR 1973 Structure-Borne Sound. Springer-Verlag, Berlin.

1 E. SKUDRZYK 1968 Simple and Complex Vibratory Systems. Pensylvania State University Press, London. 


\section{TABLE 1}

Physical properties of the experimental beam

\begin{tabular}{|l|c|}
\hline Density, $\rho\left(\mathrm{kg} / \mathrm{m}^{3}\right)$ & 7850 \\
\hline Young's modulus, $\mathrm{E}\left(\mathrm{N} / \mathrm{m}^{2}\right)$ & $207 \times 10^{9}$ \\
\hline Radius of curvature, $\mathrm{R}(\mathrm{m})$ & 1.0 \\
\hline Breadth, $\mathrm{b}(\mathrm{m})$ & 0.05 \\
\hline Depth, $\mathrm{d}(\mathrm{m})$ & $6.068 \times 10^{-3}$ \\
\hline
\end{tabular}


APPENDIX A : DERIVATION OF THE POINT MOBILITY BELOW THE RING FREQUENCY OF A 'SEMIINFINITE' BEAM WITH CONSTANT CURVATURE EXCITED BY A FORCE ACTING IN THE CIRCUMFERENTIAL DIRECTION AT THE FREE END

The mobility of a simple mass element is given by [11] as

$$
Y=\frac{-i}{\omega m} \text {. }
$$

In terms of the non-dimensional frequency, $\Omega$, this can be expressed as

$$
Y=\frac{-i R}{\Omega c_{0} m}
$$

In Figure $6(\mathrm{a})$ the modulus of the mobility at $\Omega=1.0$ is at the intersection with the point mobility of travelling extensional waves. Thus,

$$
\begin{aligned}
|Y| & =\frac{R}{\Omega c_{0} m} \\
& =8.067 \times 10^{-5}(\mathrm{~m} / \mathrm{s}) / \mathrm{N}
\end{aligned}
$$

Therefore, $m=2.414 \mathrm{R}$. But, the mass per unit length of the beam $S \rho=2.414(\mathrm{~kg})$. Hence, below the ring frequency the beams act as a mass of length equal to the radius of curvature. Further, substituting for the mass, $m=S R \rho$, into equation (A1) gives an expression for the point mobility below the ring frequency:

$$
Y=\frac{-i}{\omega S R p} \text {. }
$$

In terms of the non-dimensional frequency, $\Omega$, this becomes

$$
Y=\frac{-i}{\Omega S \rho c_{0}} .
$$


By considering the deformation of an element of a circular cylindrical shell Cremer, Heckl and Unger[10] derive on expression for total circumferential strain strain $[10$, p177] which can be written as

$$
e_{s}=\frac{1}{\left(1+\frac{z}{R}\right)}\left(\frac{W}{R}+\frac{\partial U}{\partial s}\right)
$$

where the tangential and radial displacements of the material point are $U(r, s, t)$ and $W(r, s, t)$, respectively. For small displacements of thin beams the assumptions known as 'Love's first approximation', in classical shell theory, can be made which leads to the following linear relationships between the displacemenis of a material point and components of displacement at the undeformed centre-line

$$
\begin{aligned}
& U(r, s, t)=u(R, s, t)+z \phi(s, t), \\
& W(r, s, t)=w(R, s, t),
\end{aligned}
$$

where $u$ and $w$ are the components of displacement at the centre-line in the tangential and radial directions, respectively and, $\phi$ is the rotation of the normal to the centre-line during deformation

$$
\begin{array}{lc}
\phi=\frac{\mathrm{u}}{\mathrm{R}} & -\frac{\partial \mathrm{w}}{\partial \mathrm{s}}, \\
\left(\begin{array}{l}
\text { angle of } \\
\text { curvature }
\end{array}\right) & \left(\begin{array}{l}
\text { rotational displacement } \\
\text { of straight beam }
\end{array}\right)
\end{array}
$$

and $W$ is independent of $z$ and is completely defined by the centre-line component $w$. Substituting equations (1) and (2) into the strain-displacement equation (B1) gives the following relation for total circumferential strain

$$
e_{s}=\frac{1}{\left(1+\frac{z}{R}\right)}\left(\frac{w}{R}+\frac{\partial u}{\partial s}+z \frac{\partial \phi}{\partial s}\right)
$$

This can be expressed as

$$
\mathrm{e}_{\mathrm{s}}=\frac{1}{\left(1+\frac{\mathrm{z}}{\mathrm{R}}\right)}\left(\varepsilon_{\mathrm{s}}+\mathrm{z} \beta_{\mathrm{s}}\right),
$$

where the in-plane (extensional) strain is given by

$$
\begin{aligned}
& \varepsilon_{\mathrm{s}}=\frac{\mathrm{w}}{\mathrm{R}}+\frac{\partial \mathrm{u}}{\partial \mathrm{s}}, \\
& \left(\begin{array}{l}
\text { stretching due to } \\
\text { radial displacement }
\end{array}\right)\left(\begin{array}{l}
\text { stretching due to motion } \\
\text { in circumferential direction }
\end{array}\right)
\end{aligned}
$$

and the bending strain (mid-surface change in curvature) is given by

$$
\begin{aligned}
& \beta_{s}=\frac{\partial \phi}{\partial s}, \\
& =\frac{\partial}{\partial s}\left(\frac{u}{R}-\frac{\partial w}{\partial s}\right) .
\end{aligned}
$$




\section{APPENDIX C : NOTATION}

A flexural wave amplitude

B extensional wave amplitude

E Young's modulus

$\mathrm{F}_{\mathrm{S}} \quad$ magnitude of externally applied force acting in circumferential direction

G shear modulus

I second moment of area of cross-section of beam

$\mathrm{K}$ radius of gyration

M bending moment on cross-section of beam

$\mathrm{N} \quad$ circumferential force on cross-section of beam

Q. shear force on cross-section of beam

$\mathrm{R} \quad$ radius of curvature

S cross-sectional area of beam

$\mathrm{U}$ displacement of material point in circumferential direction

W displacement of material point in radial direction

Y mobility

b breadth (width) of beam

$c_{0} \quad$ wavespeed of extensional waves in a straight bar

$\mathrm{e}_{\mathrm{s}} \quad$ total circumferential strain

h thickness of beam

k wavenumber

m mass

co-ordinate in radial direction

co-ordinate in circumferential direction

time

displacement at centre-line in circumferential direction

displacement at centre-line in radial direction

z

co-ordinate of outward pointing normal

$\Omega$

$\alpha$

non-dimensional frequency

$\beta_{\mathrm{S}} \quad$ bending strain

$\gamma_{S r} \quad$ transverse shear strain

$\varepsilon_{\mathrm{r}} \quad$ radial strain

$\varepsilon_{S} \quad$ circumferential strain

$v \quad$ Poisson's ratio

$\rho \quad$ density

$\sigma_{\mathrm{r}} \quad$ radial stress

$\sigma_{\mathrm{S}} \quad$ circumferential stress

$\sigma_{\mathrm{Sr}} \quad$ transverse shear stress

$\phi \quad$ change in slope of normal to centre-line during deformation

$\omega \quad$ radian frequency 
Figure $1 \quad$ Geometry of a curved beam

Figure 2 Sign convention and force resultants on an elemental slice of curved beam

Figure 3 Wave number versus frequency relationship for a beam with a constant radius of curvature : (*) $=$ predominantly flexural travelling wave; ' $O$ ' = predominantly flexural near field wave; ' + ' = predominantly extensional travelling wave; ' $x$ ' = predominantly extensional near field wave

Figure 4 Diagram of the tangential displacement, $U$, and radial displacement, $W$, in a 'semi-infinite' beam with curvature when excited by an harmonic force, $F_{s} e^{\text {iot }}$, acting in the circumferential direction at the free end

Figure $5 \mathrm{a} \quad$ Schematic representation of the experimental apparatus : side view

Figure $5 b \quad$ Schematic representation of the experimental apparatus : top view

Figure $6 \mathrm{a}$ Modulus of the point mobility (ie resulting extensional velocity/circumferentially acting force) of the experimental curved beam when excited by a force acting at the free end (predicted data marked with 'o' symbols)

Figure $6 \mathrm{~b} \quad$ Phase angle of the point mobility (ie resulting extensional velocity/circumferentially acting force) of the experimental curved beam when excited by a force acting at the free end (predicted data marked with 'o' symbols)

Figure 7a Modulus of the cross mobility (ie resulting flexural velocity/circumferentially acting force) of the experimental curved beam when excited by a force acting at the free end (predicted data marked with ' 0 ' symbols)

Figure $7 \mathrm{~b} \quad$ Phase angle of the cross mobility (ie resulting flexural velocity/circumferentially acting force) of the experimental curved beam when excited by a force acting at the free end (predicted data marked with 'o' symbols) 
Fig 1

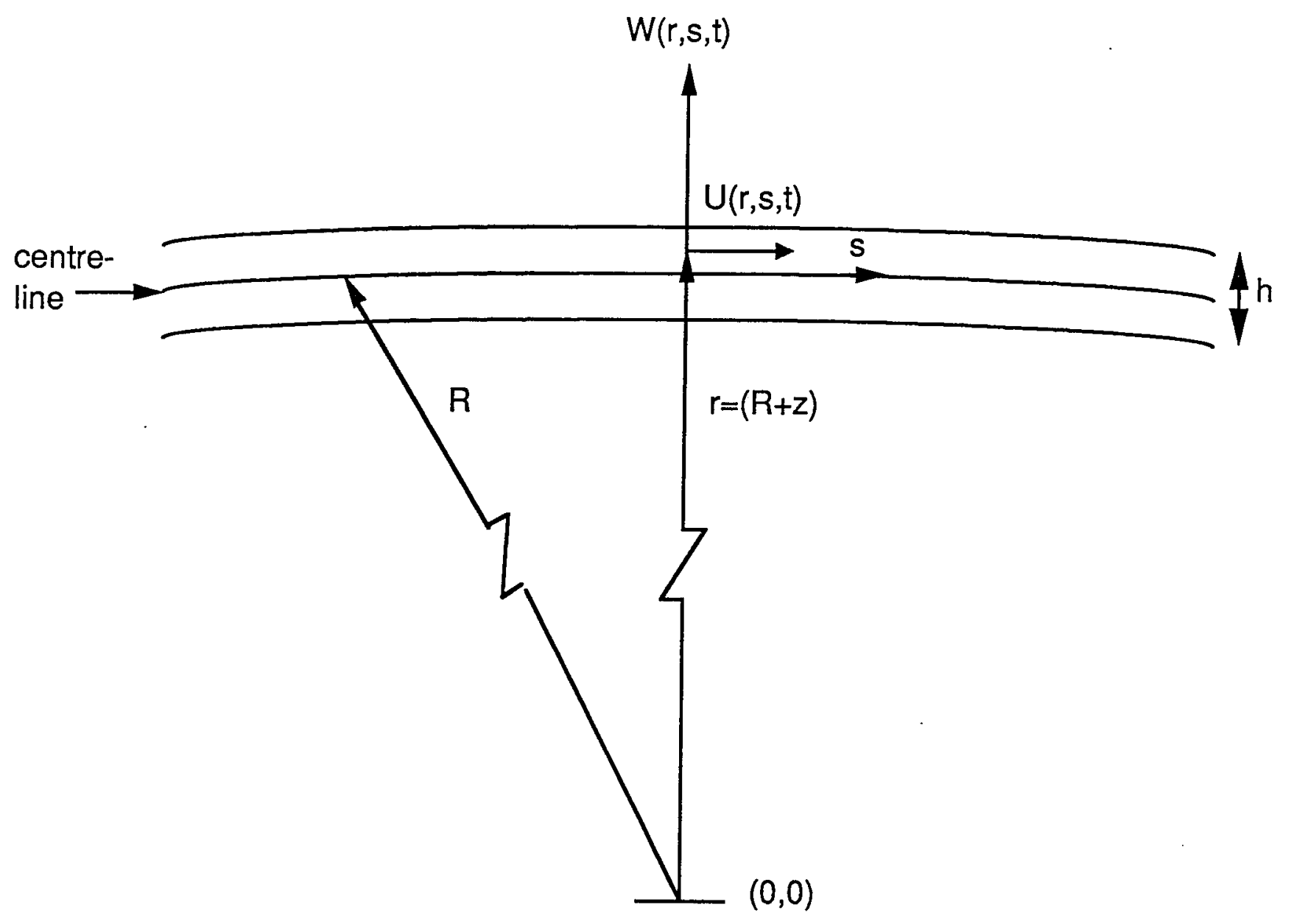



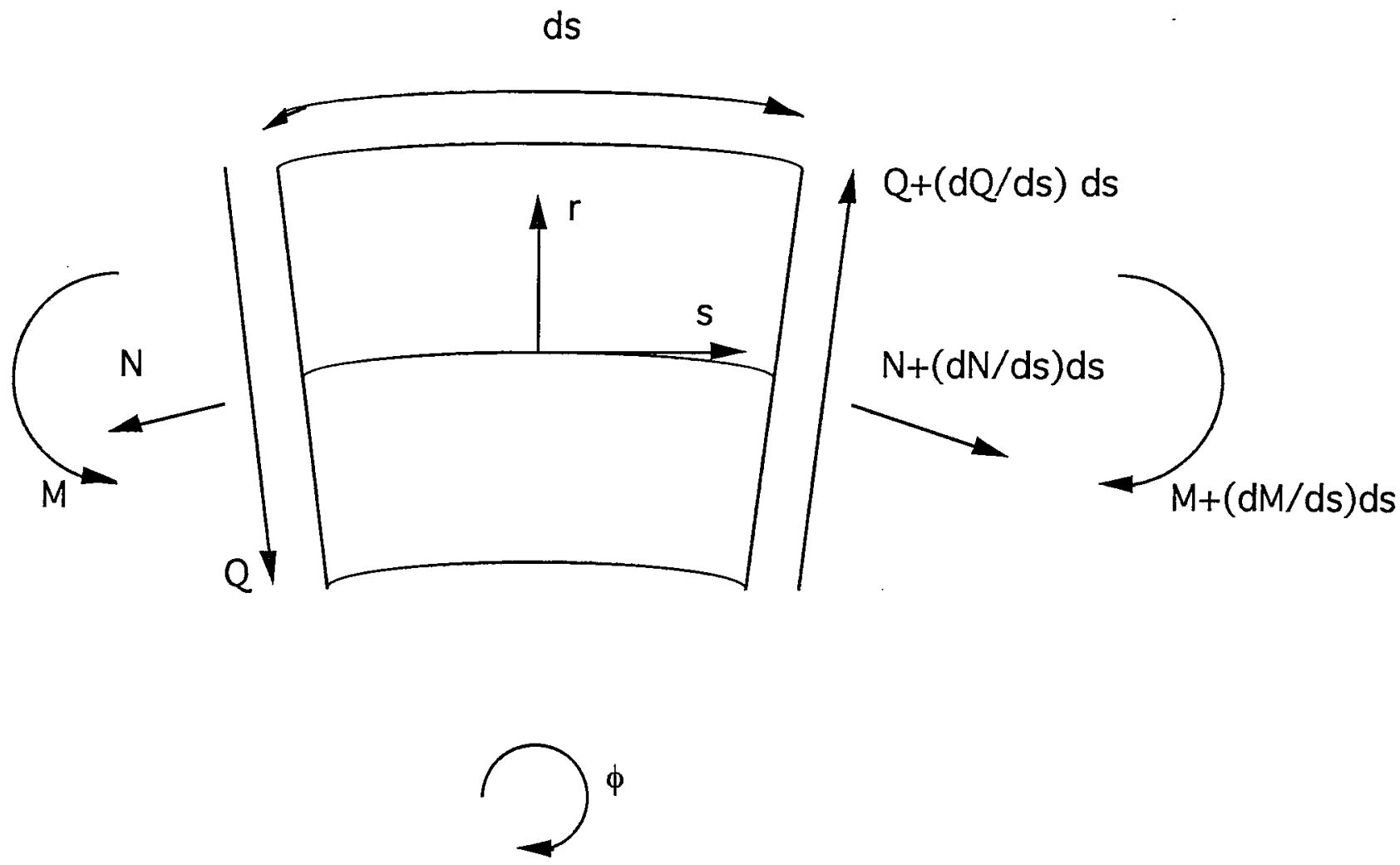


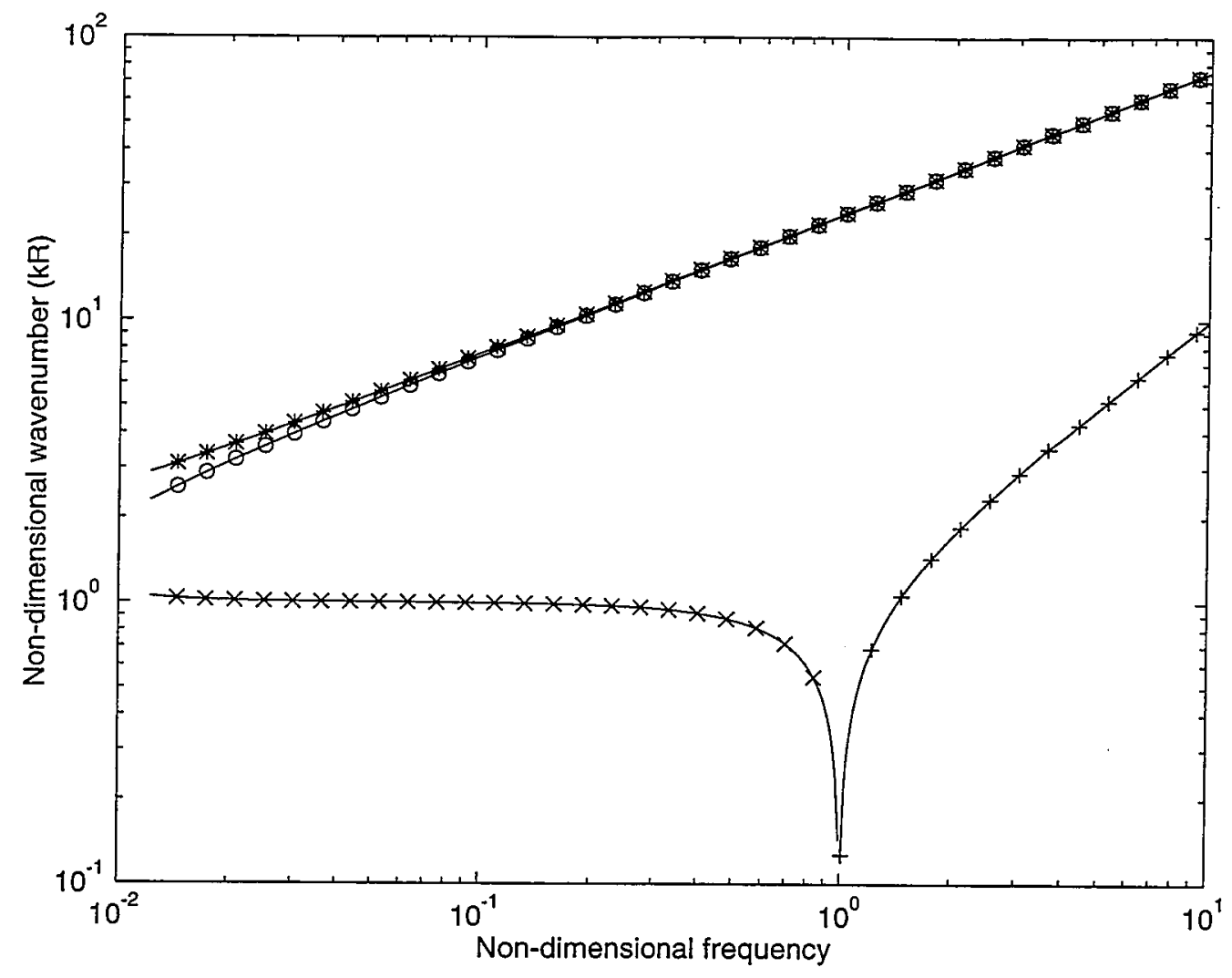




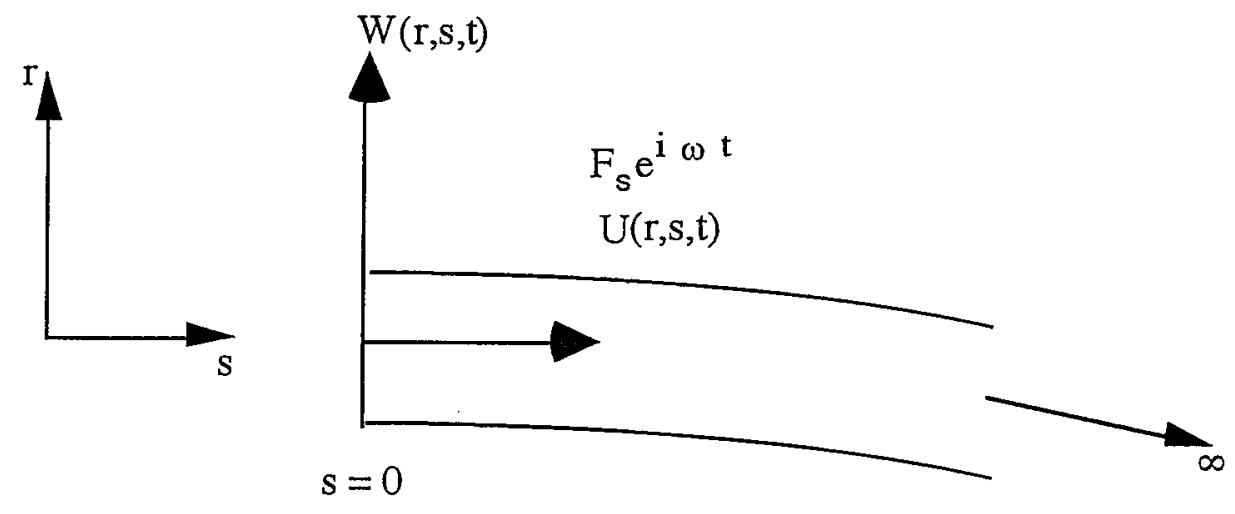




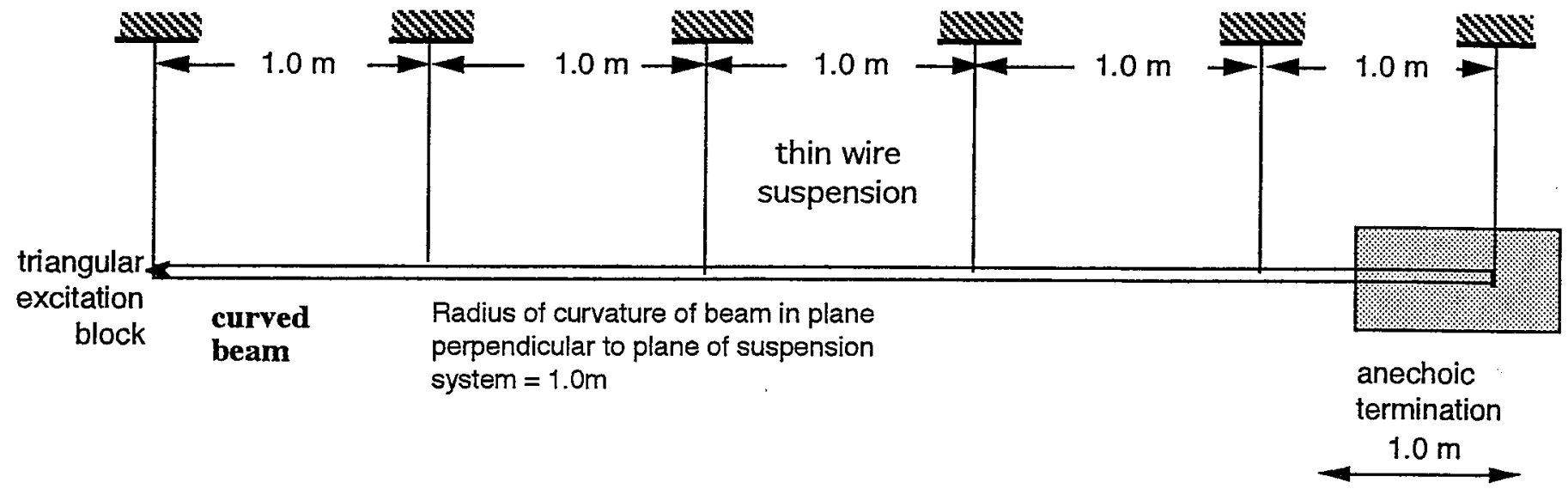


Fig 5b

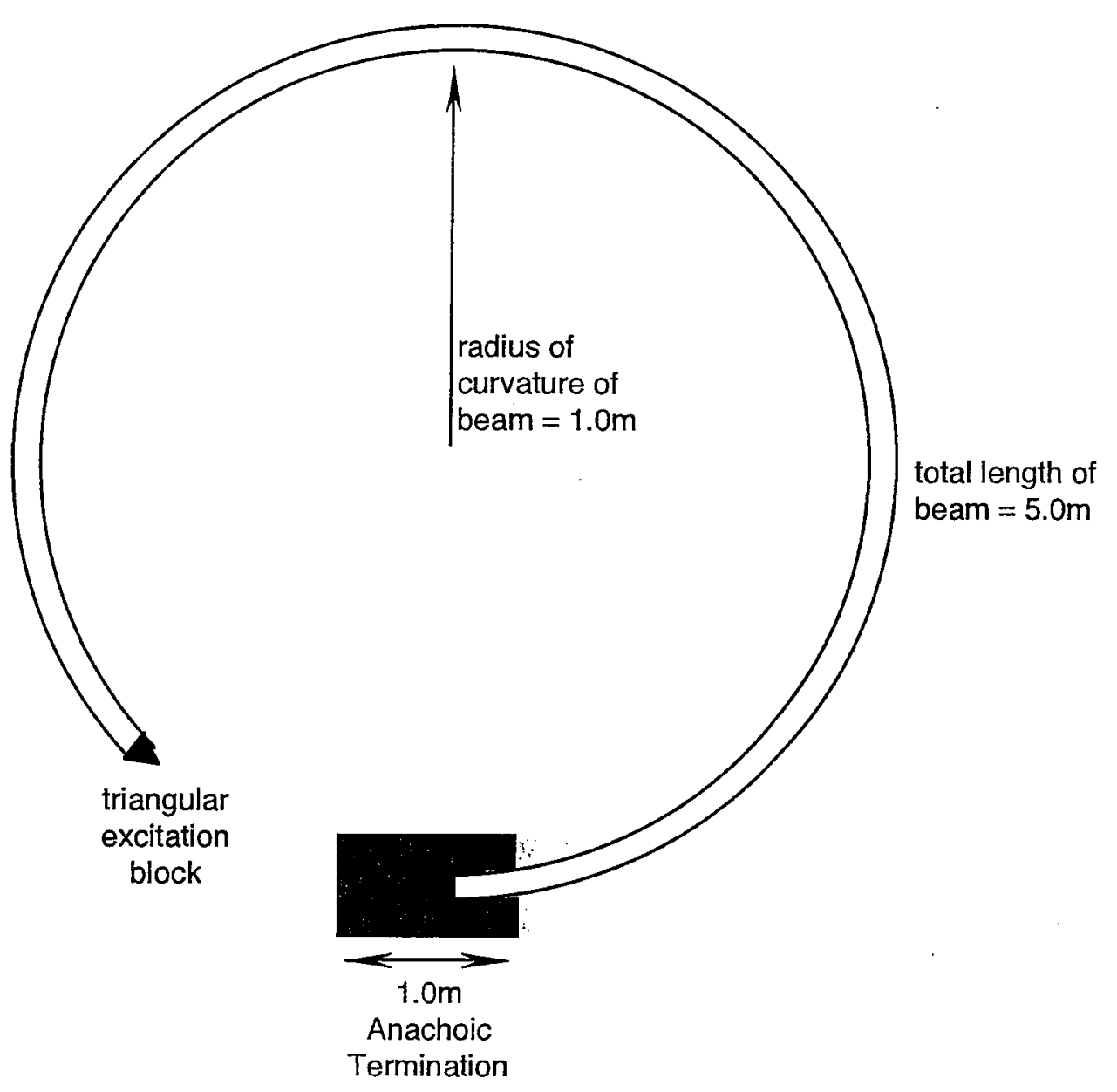


$F \lg 6(a)$

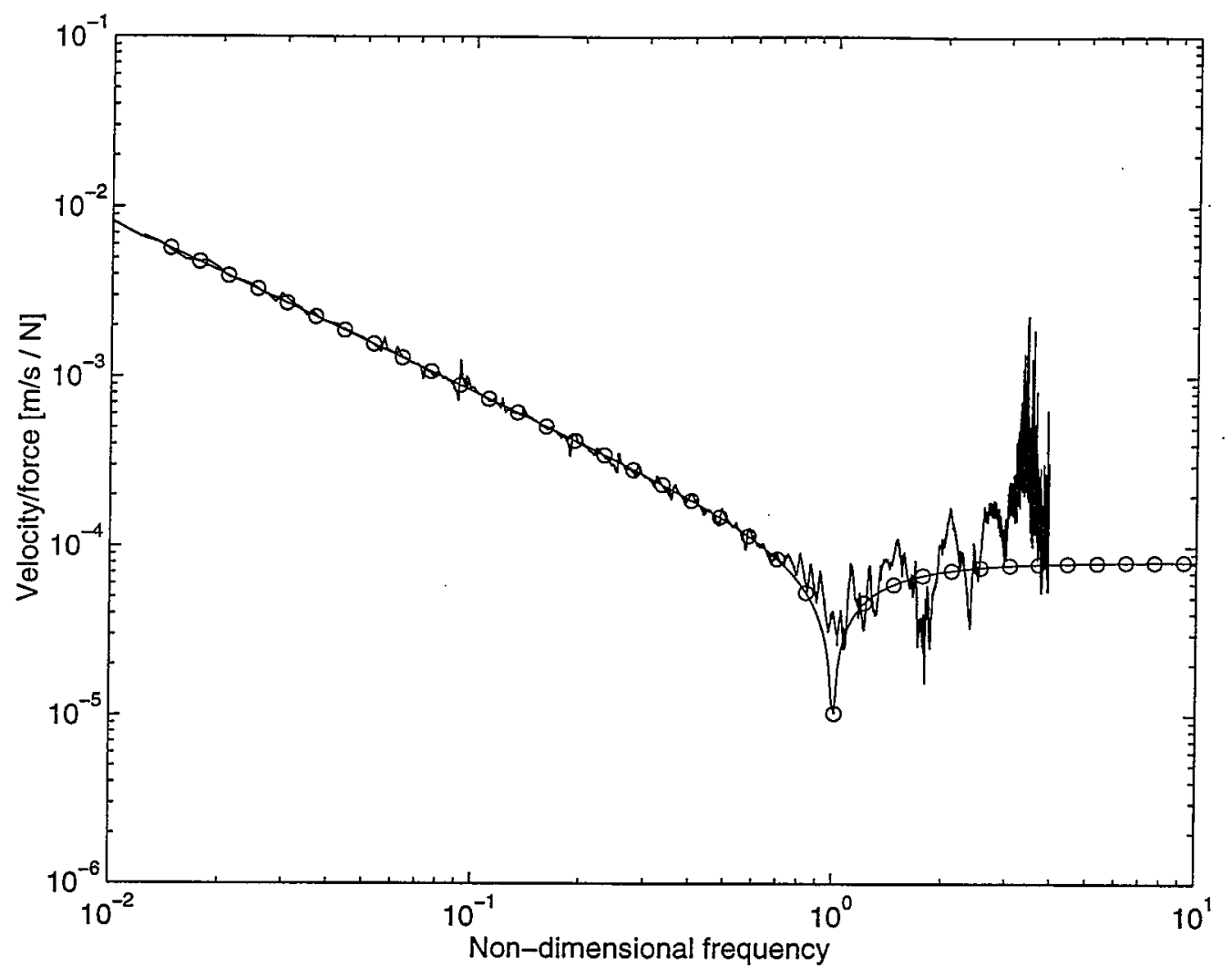


Fig $6(b)$

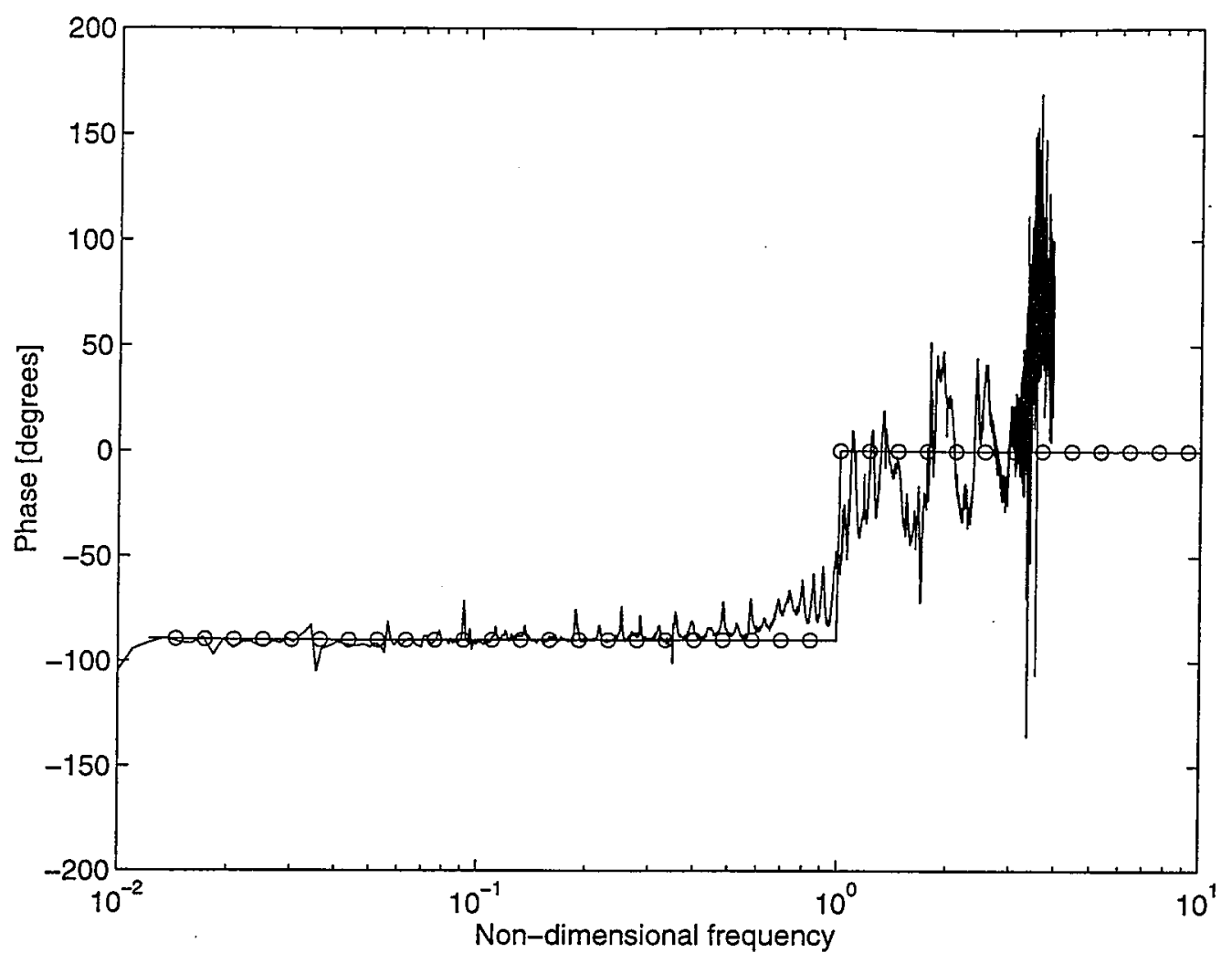


Fig 7 (a.

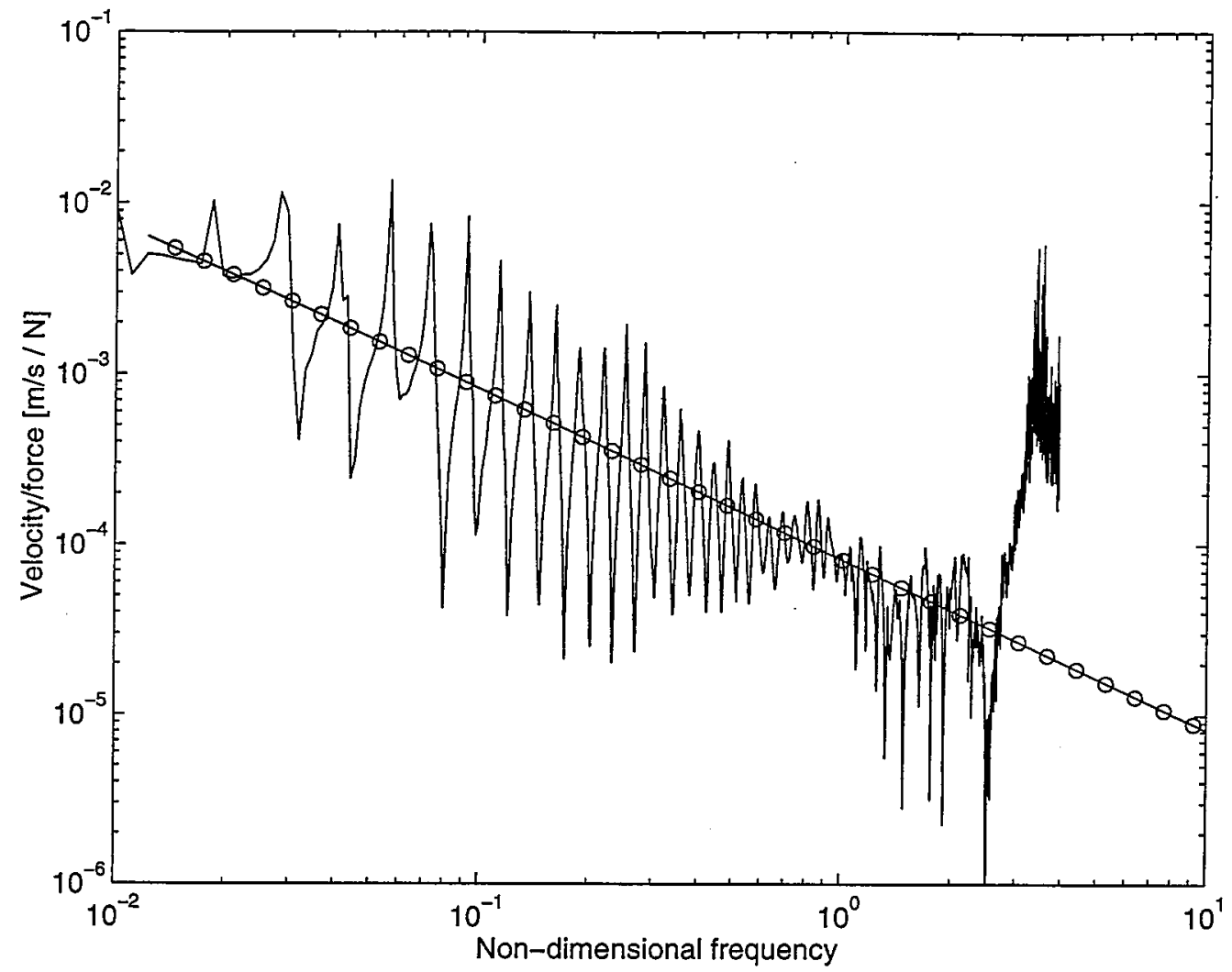




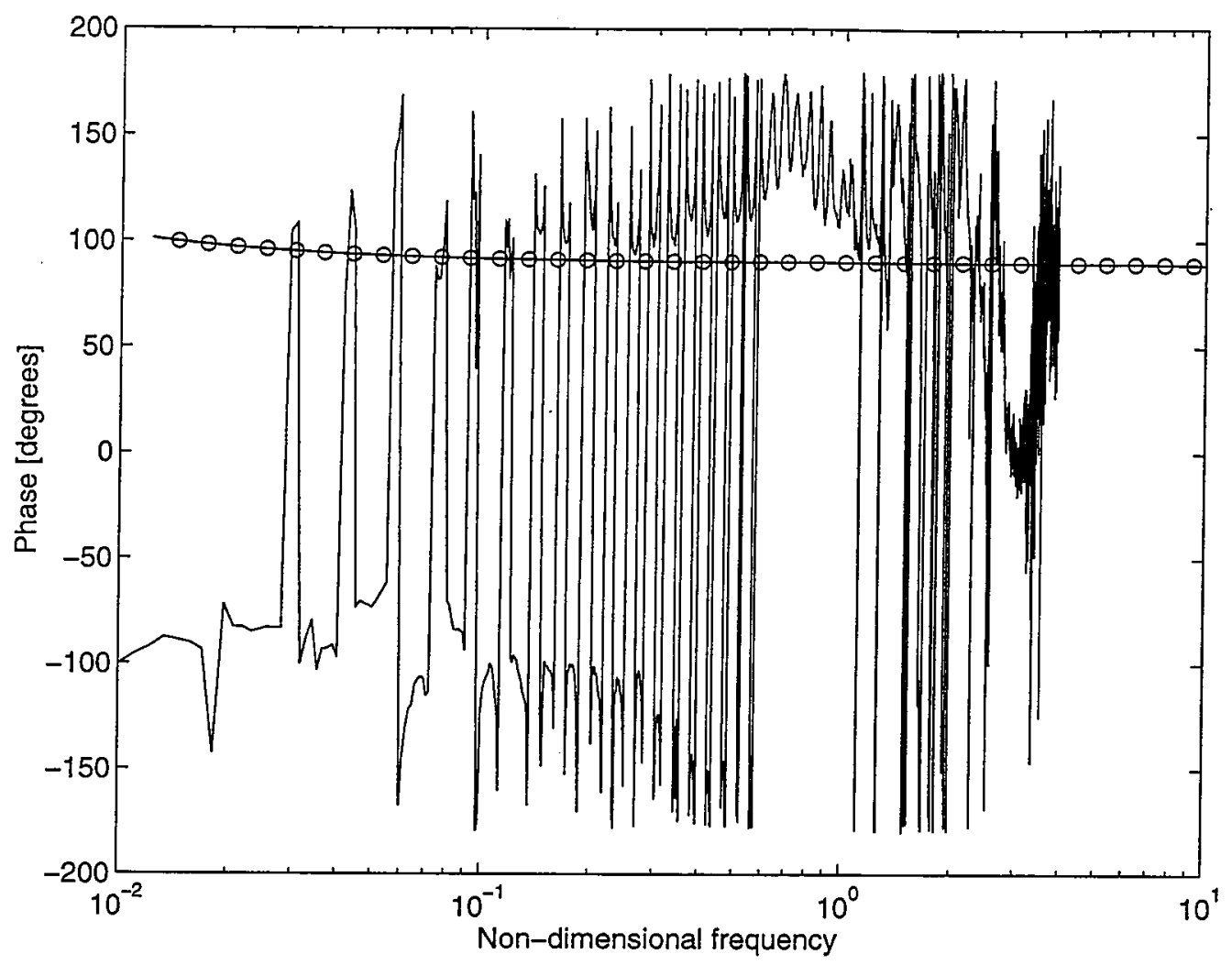

